\title{
Dual Based Procedures for Un-Capacitated Minimum Cost Flow Problem
}

\author{
Priyank Sinha, Renduchintala Raghavendra Kumar Sharma \\ Industrial and Management Engineering Department, IIT Kanpur, Kanpur, India \\ Email: spriyank@iitk.ac.in, rrks@iitk.ac.in
}

How to cite this paper: Sinha, P. and Sharma, R.R.K. (2016) Dual Based Procedures for Un-Capacitated Minimum Cost Flow Problem. American Journal of Operations Research, 6, 468-479. http://dx.doi.org/10.4236/ajor.2016.66043

Received: October 20, 2016

Accepted: November 15, 2016

Published: November 18, 2016

Copyright $\odot 2016$ by authors and Scientific Research Publishing Inc. This work is licensed under the Creative Commons Attribution International License (CC BY 4.0). http://creativecommons.org/licenses/by/4.0/

\begin{abstract}
In this article, we devise two dual based methods for obtaining very good solution to a single stage un-capacitated minimum cost flow problem. These methods are an improvement to the methods already developed by Sharma and Saxena [1]. We further develop a method to extract a very good primal solution from a given dual solution. We later demonstrate the efficacies and the significance of these methods on 150 random problems.
\end{abstract}

\section{Keywords}

Min Cost Flow, Transshipment, Dual, Primal

\section{Introduction}

Un-capacitated min cost flow problem is a special case of min cost flow problem in which arc capacities are assumed to be infinite. Weintraub [2] developed a variant of negative cycle algorithm which searched for the most negative cycle and subsequently introduced it into the feasible flow at each iteration. Later a strongly polynomial time algorithm for min cost flow was developed by Tardos [3] with a computational complexity of $\mathrm{O}\left(\mathrm{m}^{4}\right)$. Enhanced capacity scaling algorithm can be used to solve Transshipment problem with computational complexity of $\mathrm{O}(\mathrm{n} \log (\mathrm{n}) \mathrm{S}(\mathrm{n}, \mathrm{m})$ ) (Ahuja et al. [4]). Tardos [3] developed cost scaling algorithm with the computational complexity of $\mathrm{O}\left(\mathrm{n}^{3}\right.$ $\log \mathrm{n}$ ). In this algorithm, dual optimality conditions are relaxed to form e-optimality conditions. Thus the best primal based methods solve un-capacitated min cost flow problem in $\mathrm{O}\left(\mathrm{n}^{3} \log (\mathrm{n})\right)$. Recently Juman [5] has presented a heuristic with $\mathrm{O}\left(\mathrm{n}^{3}\right)$ running time to solve un-capacitated transportation problem, and is shown to perform better than VAM.

Successive shortest path algorithm was developed by Busakar and Gowan [6]. This algorithm maintains dual feasibility at each step and iteratively achieves primal feasibil- 
ity. Edmonds and Karp [7] proposed the first polynomial time algorithm by modifying the method to calculate shortest paths, to solve min cost flow problem with computational complexity of $\mathrm{O}((\mathrm{n}+\mathrm{m}) \log \mathrm{U})$. Dual simplex for network flow was first analyzed by Hegason and Kennington [8]. Plotkin and Tardos [3] improved the pivoting strategy with $\left(\mathrm{m}^{2} \log \mathrm{n}\right)$ bound over the pivoting strategy proposed by Orlin [9]. This improves the number of pivot steps required in dual simplex algorithm. This algorithm runs in $\mathrm{O}\left(\mathrm{m}^{3} \log (\mathrm{n})\right)$ time. Ali et al. [10] have demonstrated that an efficient execution of each pivot in dual based algorithm requires less iterations as compared to primal based algorithms. This holds true even for the re-optimization process. However, computational effort required per pivot may be higher. Sharma and Sharma [11] have given a new dual based procedure that has obtained solutions within $85 \%$ of the optimal.

Sharma and Saxena [1] have posed the transshipment problem differently. We use the formulation proposed by Sharma and Saxena [1]. We then modify the dual based methods developed by them to obtain better solutions with the same complexity of $\mathrm{O}\left(\mathrm{n}^{2}\right)$ and $\mathrm{O}\left(\mathrm{n}^{3}\right)$ respectively. We further devise a method to obtain a good primal solution from the dual solutions already obtained. Empirical results on the random 150 problems are given in Appendix 1.

\section{Problem Formulation}

We next present the mathematical formulation of the primal problem and dual problem respectively.

\subsection{Constants of Problem}

$D_{k}$ refers to the demand at the $k^{t h}$ demand node, while $d_{k}$ is the demand at market $k$ as a fraction of total market demand. Hence we have $d_{k}=D_{k} /\left[\sum_{k=1}^{K} D_{k}\right]$ and $\sum_{k=1}^{K} d_{k}=1$, where $K$ is the total number of demand nodes. Similarly $S_{i}$ refers to units available for transportation at the source node $i$ and $s_{i}=S_{i} / \sum_{k=1}^{K} D_{k}$. If the problem is balanced, then we have $\sum_{i=1}^{I} s_{i}=\sum_{k=1}^{K} d_{k}, I$ is the total number of supply nodes. $J$ is total number of transshipment nodes. $C 1_{i j}$ and $C 2_{j k}$ is the cost of transporting $\sum_{k=1}^{K} D_{k}$ units from node to $\mathrm{j}$ and $\mathrm{j}$ to $\mathrm{k}$ respectively.

\subsection{Decision Variables}

$x 1_{i j}$ and $x 2_{j k}$ is the number of units transported from node $i$ to node $j$ and $j$ to $k$ respectively. We also have $X 1_{i j}=x 1_{i j} /\left[\sum_{k=1}^{K} D_{k}\right]$ and $X 2_{j k}=x 2_{j k} /\left[\sum_{k=1}^{K} D_{k}\right]$.

\subsection{Primal (P)}

$$
\text { Minimize } \sum_{i=1}^{I} \sum_{j=1}^{J} X 1_{i j} C 1_{i j}+\sum_{j=1}^{J} \sum_{k=1}^{K} X 2_{j k} C 2_{j k}
$$




$$
\begin{gathered}
\text { Subject to: } \sum_{i=1}^{I} \sum_{j=1}^{J} X_{i j}=1 \quad \forall i, j \\
\sum_{j=1}^{J} \sum_{k=1}^{K} X 2_{j k}=1 \quad \forall j, k . \\
\sum_{j=1}^{J} X 1_{i j} \geq-s_{i} \quad \forall i \\
\sum_{j=1}^{J} X 2_{j k} \geq-d_{k} \quad \forall k \\
\sum_{k=1}^{K} X 2_{j k}-\sum_{i=1}^{I} X 1_{i j}=0 \quad \forall j \\
X 1_{i j}, X 2_{j k} \geq 0 \quad \forall i, j, k .
\end{gathered}
$$

In this formulation we assume flows only in the forward direction. Equation (1) ensures that entire supply is transported to meet the demand, which is valid for the balanced problem. Equation (2) ensures that the total demand is met by the supply. Equations (3) and (4) ensure that individual supply and demand constraints are satisfied, while Equation (4) ensures that no inventory is built at any transshipment node.

\subsection{Dual of the Problem (DP)}

In this section we present the dual of the problem P. We associate $V 1, V 2, U_{i}, V_{k}, W_{j}$ as the dual variables corresponding to (1), (2), (3), (4), (5) respectively. We first state the dual of the problem as DP and then divide it into two parts as DP-source and DP-sink for computational simplicity.

$D P$

$$
\begin{array}{r}
\text { Maximize }: V 1+V 2-\sum_{i=1}^{I} s_{i} U_{i}-\sum_{k=1}^{K} d_{k} V_{k} \\
\text { Subject to }: V 1-U_{i}-W_{j} \leq C 1_{i j} \quad \forall i, j \\
V 2-V_{k}+W_{j} \leq C 2_{j k} \quad \forall j, k
\end{array}
$$

$U_{i}, V_{k} \geq 0, V 1, V 2, W_{j}$ Unrestricted in sign

DP-source

$$
\begin{gathered}
\text { Maximize: } V 1-\sum_{i=1}^{I} s_{i} U_{i} \\
\text { Subject to: } V 1-U_{i}-W_{j} \leq C 1_{i j} \quad \forall i, j
\end{gathered}
$$

$U_{i} \geq 0, V 1$ and $W_{j}$ unrestricted in sign.

$D P$-sink

$$
\text { Maximize : } V 2-\sum_{k=1}^{K} d_{k} V_{k}
$$

$$
\text { Subject to: } V 2-V_{k}+W_{j} \leq C 2_{j k} \forall j, k
$$

$V_{k} \geq 0, V 2$ and $W_{j}$ unrestricted in sign. 


\section{Few Theoretical Results}

We start with development of the heuristic for the dual solution, and then move on to develop the heuristic for the primal. Computational attractiveness of these results will be demonstrated in the later sections through empirical testing. Well known dual based approaches (Orlin [9], Plotkin and Tardos [3] and Ali et al. [10]) can be used for our solution to get an advanced start while solving the transshipment problem. We begin by defining the set SPS which is as under-

SPS $=\left\{S P_{i k}: S P_{i k}\right.$ is the shortest path between $i$ and $\left.k\right\}$.

Problem (TP)

$$
\begin{gathered}
\text { Minimize }: \sum_{i=1}^{I} \sum_{k=1}^{K} X_{i k} S P_{i k} \\
\text { Subject to }: \sum_{k=1}^{K} X_{i k}=s_{i} \quad \forall i \\
\sum_{i=1}^{I} X_{i k}=d_{k} \quad \forall k
\end{gathered}
$$

and $X_{i j} \geq 0, \forall i, k$.

Theorem 1: Optimal solution of problem TP is equal to optimal solution to problem P.

Proof: Since upper value of the flow is unbounded, hence optimal flow for a pair of source node and sink node will be on $S P_{i k}$. This ensures that any further reduction in the objective value is not possible. Therefore problem TP gives the optimal solution to problem P. Hence proved.

\section{Solution Procedure}

\subsection{Heuristic to Solve Dual of the Problem (H1)}

DP-source and DP-sink are equivalent in structure to DRP1 in Sharma and Murlidhar [12]. Sharma and Murlidhar [12] have given an efficient algorithm to solve DRP1 which can be modified to solve DP-source and DP-sink.

Step 1. DP-source and DP-sink can be rewritten as under

DP-source

$$
\begin{gathered}
\text { Maximize }: V 1-\sum_{i=1}^{I} s_{i} U_{i} \\
\text { Subject to }: V 1-U_{i} \leq C 1_{i j}+W_{j}
\end{gathered}
$$

$U_{i} \geq 0, V 1$ and $W_{j}$ unrestricted in sign.

DP-sink

$$
\begin{gathered}
\text { Maximize }: V 2-\sum_{k=1}^{K} d_{k} V_{k} \\
\text { Subject to }: V 2-V_{k} \leq C 2_{j k}-W_{j}
\end{gathered}
$$

$V_{k} \geq 0, V 2$ and $W_{j}$ unrestricted in sign. 
Step 2. Find $d_{k 1}^{*}=\min _{j}\left(C 1_{i j}+W_{j}\right)$ and $d_{k 2}^{*}=\min _{j}\left(C 2_{j k}-W_{j}\right) \quad \forall$ all $i, j, k$ and $W_{j}=0$ and remove all the redundant constraints in DP-source and DP-sink (Equations (8) and (9)). In case of tie, only one equation is retained while others are eliminated. This reduces the DP-source and DP-sink to the following form:

DP-source

Maximize: $V 1-\sum_{i=1}^{I} s_{i} U_{i}$

s.t $V 1-U_{i} \leq d_{k 1}^{*} \forall i$

DP-sink

Maximize: $V 2-\sum_{k=1}^{K} d_{k} V_{k} \forall k$

s.t. $V 2-V_{k} \leq d_{k 2}^{*}$

$d_{k 1}^{*}$ and $d_{k 2}^{*}$ represent the least cost transportation route between source and transshipment node and transshipment node and sink respectively.

Step 3. We sort the values of $d_{k}^{*}$ in an increasing order and re-index such that $\left(d_{k 1}^{*}+d_{k 2}^{*}\right)_{j} \leq d_{r+1}^{*} \quad \forall r=1, \cdots, \max (i, k)$.

Step 4. Since $\sum_{k=1}^{K} d_{k}=1$ and $\sum_{i=1}^{I} s_{i}=1$, we let $V 1=d_{k 1}^{*}, U_{i}=V 1-d_{k 1}^{*}, V 2=d_{k 2}^{*}$ and $V_{k}=V 2-d_{k 2}^{*}$. Solution to the problem is given by

$\sum_{i=1}^{I} \sum_{k=1}^{K} d_{k 1}^{*}\left(\min _{j}\left(C 1_{i j}+W_{j}\right)\right)+d_{k 2}^{*}\left(\min _{j}\left(C 2_{j k}-W_{j}\right)\right)$.

We repeat the whole procedure for different increases in values of $W_{j} \forall$ all $j$ and retain the best solution.

It may be noted that when we increase/decrease the value of $W_{j} \forall j$, DP-source increases while DP-sink decreases as per the structure of DP-source and DP-sink. Actually all four possibilities are there for a general case. Our algorithm here intends to balance value of $W_{j}$ for the best trade-off possible.

Result 1: Computational complexity of $\mathrm{A} 1$ is $\mathrm{O}\left(\mathrm{n}^{2}\right)$.

Proof: Complexity of algorithm is dominated by step 2 which can be solved in $\mathrm{O}(\mathrm{n} 2)$ time.

\subsection{Heuristic to Solve Dual of the Problem (H2)}

In the previous algorithm, we tinkered with value of $W_{j}$ along $S P_{i k}$. There is no reason as to why we should not tinker with the values of $U_{i}, V_{k}, W_{j}$ along $S P_{i k}$. $\operatorname{Min}\left(C 1_{i j}-V 1+U_{i}+W_{j}\right)$ and $\operatorname{Min}\left(C 2_{j k}-V 2+V_{k}-W_{j}\right)$ are achieved simultaneously along $S P_{i k}$ in this method. $\left(C 1_{i j}-V 1+U_{i}+W_{j}\right)$ and $\left(C 2_{j k}-V 2+V_{k}-W_{j}\right)$ are defined as source slack and sink slack respectively $\left(S_{s o}, S_{s i}\right)$ in the later sections. Next we describe this heuristic in detail.

Step 0: Set $W_{j}=0 \forall j=1, \ldots, J$.

Step 1: Compute max_value of DP_source and DP_sink, set current_best_DP = objective function value of (DP source + DP-sink), set $j=0$.

Step 2: $j=j+1$; if $j>J$ then stop or else go to step 3 . 
Step 3: Increase value of $W_{j}$ in steps and compute for each value of $W_{j}$ max_value of DP-source and DP-sink.

Step 4: Set current_DP = objective function value of (DP-source + DP-sink).

If current_DP > current_best_DP then current_best_DP = current_DP go to step 3, else go to step 2.

Result 2: Heuristic 2 runs in $\mathrm{O}\left(\mathrm{n}^{3}\right)$ time.

Proof. Complexity of the step is heuristic is dominated by step 3 which can be completed in $\mathrm{O}\left(\mathrm{n}^{3}\right)$ steps.

\subsection{Development of the Heuristic to Obtain a Good Primal Solution (H3)}

In this section we will develop a primal heuristic by utilizing the complimentary slackness condition. This heuristic extracts a good primal solution from a good dual solution by utilizing complimentary slackness condition. Let us denote the solution of DP by $V_{s S o,}, V_{s S i} U=\left\{U_{i}, \forall i=1, \cdots, I\right\}, V=\left\{V_{k}, \forall k=1, \cdots, K\right\}$. We further define $l_{p}$ $j=1, \cdots, J$ and $l_{k}, k=1, \cdots, k$ as $l_{j}=\left\{j: \operatorname{Cssot}_{i j}+W_{j}=d_{k 1}^{*}\right\}$ and $l_{k}=\left\{j: C t s i_{j k}-W_{j}=d_{k 2}^{*}\right\}$ where $d_{k 1}^{*}=\min _{j}\left(C s o t_{i j}+W_{j}\right)$ and $d_{k 2}^{*}=\min _{j}\left(C t s i_{j k}-W_{j}\right)$. Slack $S_{s o}$ and $S_{s i}$ is defined as following:

$S_{s o}=\left|\operatorname{Csot}_{i j}-V_{s s o}+U_{i}+W_{j}\right|$ and $S_{s i}=\left|C t s i_{j k}-V_{s s i}+V_{k}-W_{j}\right|$. If $S_{s o}=0$ and $S_{s i}=0$, then $X_{i j} \geq 0$ and $X_{j k} \geq 0 . X_{i j}$ and $X_{j k}$ can assume a positive value if for the corresponding $i$ and $k$, we have $i \in l_{j}$ and $k \in l_{k}$. According to the complimentary slackness condition, $X_{i j}=0$ and $X_{j k}=0$ when $i \neq l_{j}$ and $k \neq l_{k}$. Let $S_{s o}$ and $S_{s i}$ be the source and sink slacks respectively, $S P_{i k}=\min _{j}\left(\sum_{i, j} S_{s o}+\sum_{j, k} S_{s i}\right)$ and $D N_{i k}=S P_{i k} \times X_{i k} D N_{i k}$ is then referred to as deviation number. If $S P_{i k}=0$, then we can send a positive flow along this arc without violating the Complimentary slackness property. However if $S P_{i k}>0$, then flow along this has to be zero if complimentary slackness property is not to be violated. As we are working with good dual solution (and not optimal dual solution), we may have to send positive flow along a path $(i, k)$ even if $S P_{i k}>0$. But the heuristic so described tries to minimize $D N_{i k}$ and hence keep complementary slackness violations as low as possible to get good primal solution. If at the end of execution of algorithm $D N$ $=0$, then we have the optimal primal solution. In this way $D N_{i k}$ is similar to Kilter number (ref OUT-OF-KILTER algorithm (a primal dual approach) [13] for solving general min-cost-flow problem).

We find shortest path from every source node ' $i$ ' to every sink node ' $k$ ' using these slacks as weights, and then make the allocations according to shortest path available. Detailed heuristic is described as under.

Step 0: $X_{i j}=X_{j k}=0, S_{i 1 k 1}=0 \forall$ all $i, j$ and $k$.

Step 1: Compute $S_{i k}=S_{s o}+S_{s i} \forall$ all $j$ and particular $i$ and $k$.

$$
S_{s o}=\left|C s o t_{i j}-V_{s s o}+U_{i}+W_{j}\right|, \quad S_{s i}=\left|C t s i_{j k}-V_{s s i}+V_{k}-W_{j}\right|
$$

And $S_{i k}=S_{s o^{+}}+S_{s i} \forall j^{\prime} \neq j$ for the same $i, k$. 
If $S_{i k},<S_{i k}$ then $S_{i 1 k 1}=S_{i k}$, Repeat the step $\forall i$, jand $k$.

Step 2: Find $i$ and $k: d_{k}>0$ and $b_{i}>0$.

If $S_{i 1 k 1}<S_{i 2 k 2}: i 1 \neq i 2$ or $k 1 \neq k \forall$ all $i$ and $k$ then $X_{i j}=X_{j k}=S_{i l k 1}=\min \left(b_{p}, d_{k}\right)=a^{*}, b_{i}=$ $b_{i}-a^{*}, d_{k}=d_{k}-a^{*}$.

Step 3: Stop.

Result 3: Heuristic $\mathrm{H} 2$ runs in $\mathrm{O}(\mathrm{n} 2)$ time.

Proof: Complexity is dominated by the step 1 which is sorting and can be solved in $\mathrm{O}\left(\mathrm{n}^{2}\right)$.

\section{Results and Discussion}

We have solved 150 random problems of varying sizes using methods proposed in this article and the ones developed by Sharma and Saxena [1]. We performed one tail paired-test and F-test on the results. Results of paired t-test are as follows. In terms of duality gap, Subroutine $\mathrm{S} 3\left(\mathrm{O}\left(\mathrm{n}^{3}\right)\right)$ performs better than subroutine $\mathrm{S} 2(\mathrm{O}(\mathrm{n} 2))$ with the statistical significance of 0.00722 (p-value) in Sharma and saxena [1]. Similarly in terms of duality gap, $\mathrm{H} 2\left(\mathrm{O}\left(\mathrm{n}^{3}\right)\right)$ in this paper performs better than $\mathrm{H} 1\left(\mathrm{O}\left(\mathrm{n}^{2}\right)\right)$ with a statistical significance of 0.000419 (p-value). $\mathrm{H} 2\left(\mathrm{O}\left(\mathrm{n}^{3}\right)\right)$ in this article performs better than $\mathrm{S} 3\left(\mathrm{O}\left(\mathrm{n}^{2}\right)\right)$ form Sharma and saxena [1] with a statistical significance of 2.94E-15. For F-test, F-statistic was calculated to be 58.31 as against the f-critical value of 2.62. $\mathrm{P}$-value was calculated to be $4.05 \mathrm{E}-32$. In terms of computational time, no significant difference is registered between these methods, however methods in this paper perform slightly better than those proposed in Sharma and saxena [1]. This is largely due to the fact that we calculate shortest path between the source nodes and sink nodes in contrast to shortest path individually between source and transshipment nodes and transshipment nodes and sink nodes respectively. This method is better computationally.

\section{Conclusion}

In this work we have developed computationally efficient dual based method to achieve good solution to un-capacitated transshipment problem. As stated earlier, available primal and dual based approaches are capable of solving un-capacitated transshipment problem in $\mathrm{O}\left(\mathrm{n}^{3} \log (\mathrm{n})\right)$ and $\mathrm{O}\left(\mathrm{m}^{3} \log (\mathrm{n})\right)$ time respectively. Computational complexities of $\mathrm{H} 1, \mathrm{H} 2$ and $\mathrm{H} 3$ are $\mathrm{O}\left(\mathrm{n}^{2}\right), \mathrm{O}\left(\mathrm{n}^{3}\right)$ and $\mathrm{O}\left(\mathrm{n}^{2}\right)$ respectively. Later we intend to extend this work to General Minimum Cost Flow Problem which would have additional capacity constraints on the arcs.

\section{References}

[1] Sharma, R.R.K. and Saxena, A. (2002) Dual Based Procedures for the Special Case of Transshipment Problem. Operation Research, 39, 177-188.

[2] Weintraub, A. (1974) A Primal Algorithm to Solve Network Flow Problems with Convex Costs. Management Science, 21, 87-97. https:/doi.org/10.1287/mnsc.21.1.87

[3] Plotkin, S.A. and Tardos, E. (1990) Improved Dual Network Simplex. Proceedings of the 1st Annual ACM-SIAM Symposium on Discrete Algorithms, Society for Industrial and Applied Mathematics, San Francisco, 22-24 January 1990, 367-376. 
[4] Ahuja, R.K. (1993) Network Flows. PhD Thesis, Technische Hochshule Darmstadt, Darmstadt.

[5] Juman, Z.A.M.S. and Hoque, M.A. (2015) An Efficient Heuristic to Obtain a Better Initial Feasible Solution to the Transportation Problem. Applied Soft Computing, 34, 813-826.

https:/doi.org/10.1016/j.asoc.2015.05.009

[6] Busaker, R.G. and Gowen, P.J. (1961) A Procedure for determining Minimal-Cost Flow Network Patterns. Tech. Rep. ORO-15, Operational Research Office, Johns Hopkins University, Baltimore.

[7] Edmonds, J. and Karp, R.M. (1972) Theoretical Improvements in Algorithmic Efficiency for Network Flow Problems. Association for Computing Machinery Journal, 19, 248-264.

[8] Helgason, R.V. and Kennington, J.L. (1977) An Efficient Procedure for Implementing a Dual Simplex Network Flow Algorithm. AIIE Transactions, 9, 63-68.

https:/doi.org/10.1080/05695557708975122

[9] Orlin, J.B. (1984) Genuinely Polynomial Simplex and Non-Simplex Algorithms for Minimum Cost Problems. Technical Report 1615-84, Sloan School of Management, MIT, Cambridge, MA.

[10] Ali, A.I., Padman, R. and Thiagarajan, H. (1989) Dual Algorithms for Pure Network Problems. Operations Research, 37, 159-171. https:/doi.org/10.1287/opre.37.1.159

[11] Sharma, R.R.K. and Sharma, K.D. (2000) A New Dual Based Procedure for the Transportation Problem. European Journal of Operational Research, 122, 611-624.

https:/doi.org/10.1016/S0377-2217(99)00081-8

[12] Sharma, R.R.K. and Muralidhar, A. (2009) A New Formulation and Relaxation of the Simple Plant Location Problem. Asia-Pacific Journal of Operational Research, 26, 1-11. https:/doi.org/10.1142/S0217595909002122

[13] Clasen, R.J. (1968) The Numerical Solution of Network Problems Using the Out-of-Kilter Algorithm. No. RM-5456-PR. RAND CORP Santa Monica. 


\section{Appendix 1}

Results of 150 Problems.

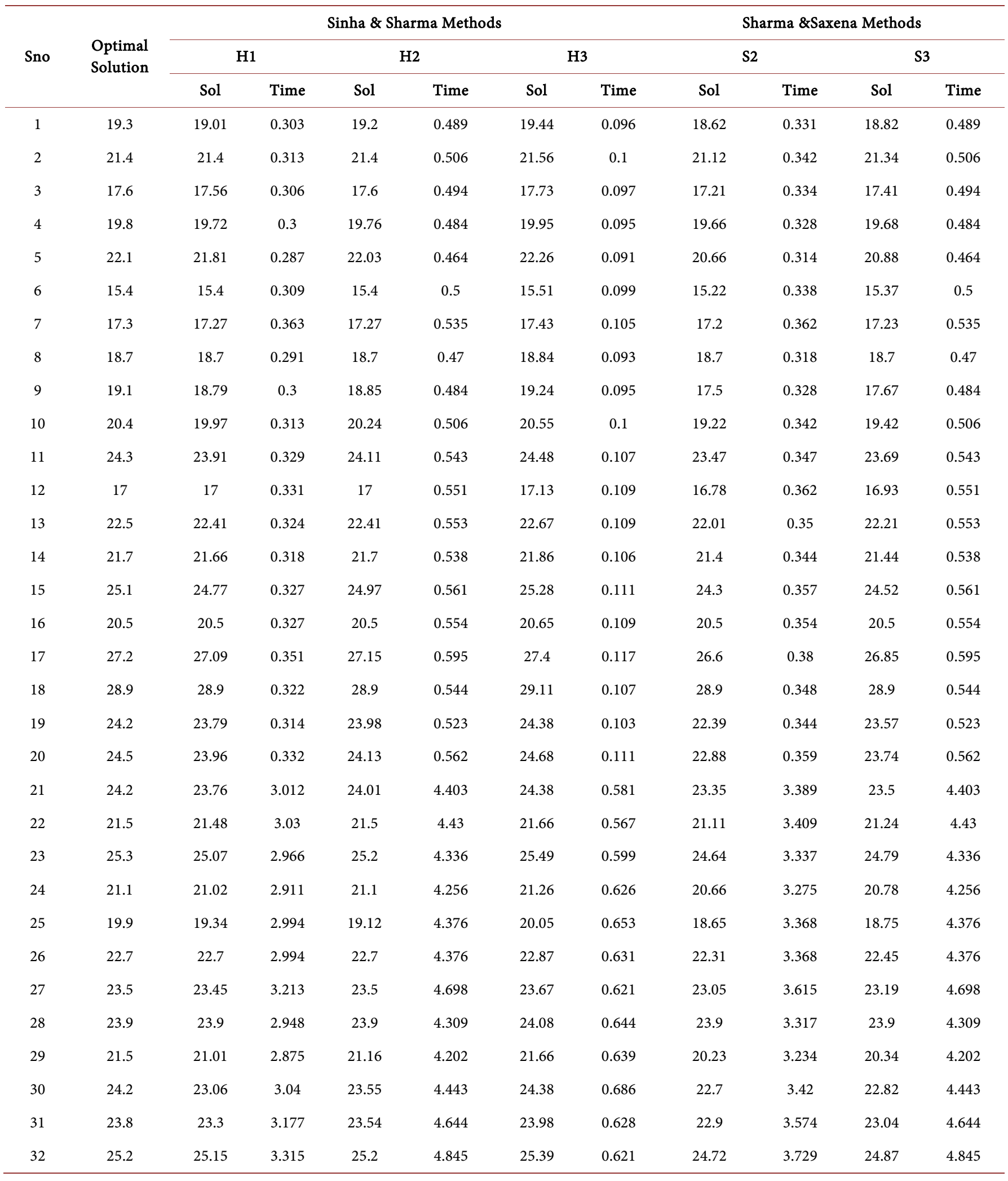


Continued

\begin{tabular}{|c|c|c|c|c|c|c|c|c|c|c|c|}
\hline 33 & 27.4 & 27.1 & 3.204 & 27.1 & 4.684 & 27.6 & 0.648 & 26.63 & 3.605 & 26.8 & 4.684 \\
\hline 34 & 21.8 & 21.58 & 3.149 & 21.63 & 4.603 & 21.96 & 0.547 & 21.21 & 3.543 & 21.34 & 4.603 \\
\hline 35 & 24.6 & 24.08 & 3.268 & 24.33 & 4.777 & 24.78 & 0.565 & 23.67 & 3.677 & 23.81 & 4.777 \\
\hline 36 & 22.9 & 22.9 & 3.241 & 22.9 & 4.737 & 23.07 & 0.552 & 22.56 & 3.646 & 22.69 & 4.737 \\
\hline 37 & 23.8 & 23.54 & 3.479 & 23.78 & 5.085 & 23.98 & 0.541 & 23.13 & 3.914 & 23.28 & 5.085 \\
\hline 38 & 26.1 & 26.1 & 3.186 & 26.1 & 4.657 & 26.29 & 0.518 & 26.1 & 3.584 & 26.1 & 4.657 \\
\hline 39 & 25.3 & 24.69 & 3.149 & 24.95 & 4.603 & 25.49 & 0.558 & 24.26 & 3.543 & 24.41 & 4.603 \\
\hline 40 & 24.7 & 23.98 & 3.287 & 24.23 & 4.805 & 24.88 & 0.655 & 23.56 & 3.698 & 23.71 & 4.805 \\
\hline 41 & 25.5 & 25.17 & 2.774 & 25.42 & 4.055 & 25.69 & 0.525 & 24.74 & 3.121 & 24.89 & 4.055 \\
\hline 42 & 21.2 & 21.2 & 2.866 & 21.2 & 4.188 & 21.36 & 0.541 & 20.88 & 3.224 & 21.01 & 4.188 \\
\hline 43 & 21.3 & 21.3 & 2.802 & 21.3 & 4.095 & 21.46 & 0.565 & 20.94 & 3.152 & 21.07 & 4.095 \\
\hline 44 & 23.2 & 23.15 & 2.747 & 23.15 & 4.015 & 23.37 & 0.597 & 22.76 & 3.09 & 22.9 & 4.015 \\
\hline 45 & 20.7 & 20.7 & 2.628 & 20.7 & 3.841 & 20.85 & 0.955 & 20.12 & 2.956 & 20.24 & 3.841 \\
\hline 46 & 21.6 & 21.6 & 2.829 & 21.6 & 4.135 & 21.76 & 0.923 & 21.28 & 3.183 & 21.41 & 4.135 \\
\hline 47 & 22.8 & 22.62 & 3.324 & 22.71 & 4.858 & 22.97 & 0.907 & 22.41 & 3.739 & 22.55 & 4.858 \\
\hline 48 & 20.8 & 20.7 & 2.664 & 20.76 & 3.894 & 20.95 & 0.941 & 20.49 & 2.997 & 20.61 & 3.894 \\
\hline 49 & 22.9 & 22.58 & 2.747 & 22.83 & 4.015 & 23.07 & 0.934 & 22.19 & 3.09 & 22.33 & 4.015 \\
\hline 50 & 22.5 & 22.07 & 2.866 & 22.3 & 4.188 & 22.67 & 1.002 & 21.69 & 3.224 & 21.83 & 4.188 \\
\hline 51 & 23.8 & 23.09 & 3.03 & 23.32 & 4.43 & 23.98 & 0.918 & 22.68 & 3.409 & 22.82 & 4.43 \\
\hline 52 & 22.1 & 21.92 & 3.132 & 22.01 & 4.577 & 22.26 & 0.907 & 21.55 & 3.523 & 21.68 & 4.577 \\
\hline 53 & 22.5 & 22.12 & 3.058 & 22.34 & 4.47 & 22.67 & 0.947 & 21.74 & 3.44 & 21.87 & 4.47 \\
\hline 54 & 23.1 & 23.1 & 3.003 & 23.1 & 4.389 & 23.27 & 0.799 & 23.1 & 3.378 & 23.1 & 4.389 \\
\hline 55 & 21.8 & 21.34 & 2.875 & 21.54 & 4.202 & 21.96 & 0.825 & 20.14 & 3.234 & 20.25 & 4.202 \\
\hline 56 & 26.7 & 26.51 & 3.094 & 26.59 & 4.523 & 26.9 & 0.807 & 26.06 & 3.481 & 26.22 & 4.523 \\
\hline 57 & 25.3 & 25.27 & 3.315 & 25.27 & 4.845 & 25.49 & 0.791 & 24.84 & 3.729 & 25 & 4.845 \\
\hline 58 & 27.6 & 27.6 & 2.911 & 27.6 & 4.256 & 27.8 & 0.757 & 27.6 & 3.275 & 27.6 & 4.256 \\
\hline 59 & 27.1 & 26.5 & 3.003 & 26.8 & 4.389 & 27.3 & 0.815 & 25.07 & 3.378 & 25.2 & 4.389 \\
\hline 60 & 24.9 & 24.63 & 3.132 & 23.85 & 4.577 & 25.08 & 0.957 & 23.18 & 3.523 & 23.38 & 4.577 \\
\hline 61 & 25.5 & 24.76 & 3.395 & 25.02 & 4.961 & 25.69 & 0.767 & 24.33 & 3.819 & 24.48 & 4.961 \\
\hline 62 & 26.4 & 26.4 & 3.492 & 26.4 & 5.105 & 26.59 & 0.791 & 25.74 & 3.929 & 25.9 & 5.105 \\
\hline 63 & 24.6 & 24.18 & 3.458 & 24.43 & 5.054 & 24.78 & 0.825 & 23.76 & 3.89 & 23.91 & 5.054 \\
\hline 64 & 21.5 & 21.31 & 3.365 & 21.39 & 4.919 & 21.66 & 1.565 & 20.94 & 3.786 & 21.07 & 4.919 \\
\hline 65 & 23.9 & 23.25 & 3.51 & 23.49 & 5.13 & 24.08 & 1.574 & 22.85 & 3.949 & 22.99 & 5.13 \\
\hline 66 & 23.2 & 23.2 & 3.464 & 23.2 & 5.062 & 23.37 & 1.541 & 22.92 & 3.897 & 23.06 & 5.062 \\
\hline 67 & 24.8 & 24.38 & 3.718 & 24.63 & 5.435 & 24.98 & 1.513 & 23.96 & 4.183 & 24.11 & 5.435 \\
\hline 68 & 24.7 & 24.7 & 3.4 & 24.7 & 4.97 & 24.88 & 1.555 & 24.7 & 3.825 & 24.7 & 4.97 \\
\hline 69 & 22.6 & 22.08 & 3.302 & 21.92 & 4.827 & 22.77 & 1.555 & 21.33 & 3.715 & 21.45 & 4.827 \\
\hline 70 & 22.1 & 21.48 & 3.51 & 21.64 & 5.13 & 22.26 & 1.67 & 20.71 & 3.949 & 20.82 & 5.13 \\
\hline 71 & 21.6 & 21.41 & 6.218 & 21.56 & 7.941 & 21.76 & 1.532 & 21.06 & 6.599 & 21.19 & 7.941 \\
\hline 72 & 22.8 & 22.21 & 6.256 & 22.44 & 7.989 & 22.97 & 1.494 & 21.84 & 6.639 & 21.98 & 7.989 \\
\hline
\end{tabular}


Continued

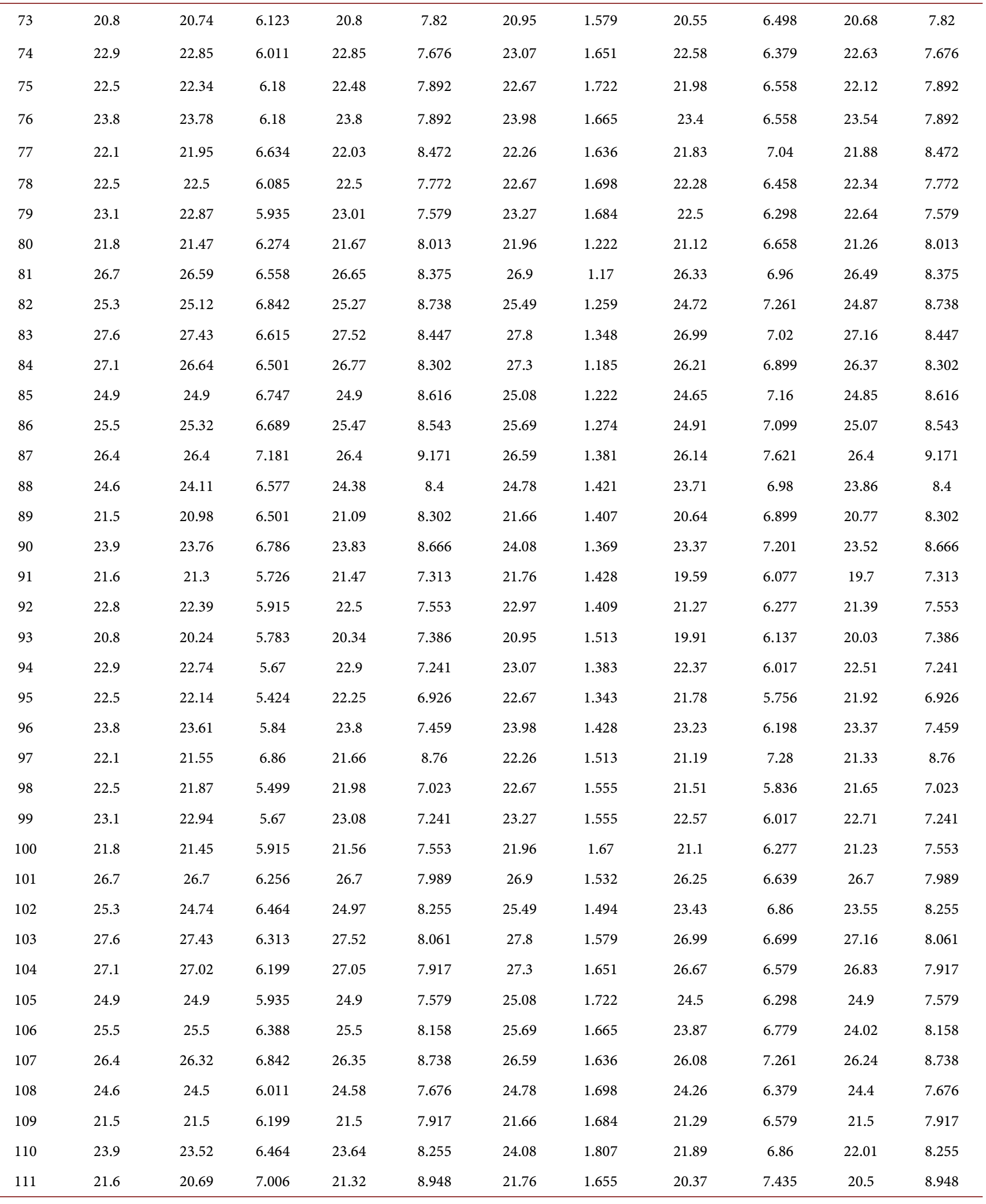




\section{Continued}

\begin{tabular}{|c|c|c|c|c|c|c|c|c|c|c|c|}
\hline 112 & 22.8 & 22.37 & 7.211 & 22.48 & 9.209 & 22.97 & 1.636 & 22 & 7.652 & 22.14 & 9.209 \\
\hline 114 & 22.9 & 22.74 & 6.947 & 22.88 & 8.872 & 23.07 & 1.441 & 22.37 & 7.372 & 22.51 & 8.872 \\
\hline 115 & 22.5 & 22.37 & 7.245 & 22.43 & 9.253 & 22.67 & 0.67 & 22.01 & 7.689 & 22.14 & 9.253 \\
\hline 116 & 23.8 & 23.4 & 7.149 & 23.51 & 9.13 & 23.98 & 0.651 & 23.01 & 7.587 & 23.16 & 9.13 \\
\hline 118 & 22.5 & 22.43 & 7.019 & 22.46 & 8.964 & 22.67 & 1.225 & 21.98 & 7.449 & 22.12 & 8.964 \\
\hline 119 & 23.1 & 22.98 & 6.817 & 23.03 & 8.706 & 23.27 & 1.233 & 22.87 & 7.234 & 22.89 & 8.706 \\
\hline 120 & 21.8 & 21.45 & 7.245 & 21.56 & 9.253 & 21.96 & 1.207 & 21.02 & 7.689 & 21.15 & 9.253 \\
\hline 121 & 26.7 & 26.49 & 6.501 & 26.46 & 6.746 & 26.9 & 1.185 & 26.38 & 6.61 & 26.54 & 6.61 \\
\hline 125 & 24.9 & 24.15 & 6.577 & 24.9 & 6.825 & 25.08 & 1.199 & 24.6 & 6.688 & 23.46 & 6.688 \\
\hline 126 & 25.5 & 25.3 & 6.501 & 25.19 & 6.746 & 25.69 & 1.17 & 25.25 & 6.61 & 25.02 & 6.61 \\
\hline 127 & 26.4 & 25.95 & 6.786 & 26.29 & 7.042 & 26.59 & 1.236 & 25.71 & 6.9 & 25.45 & 6.9 \\
\hline 128 & 24.6 & 24.6 & 5.726 & 24.58 & 5.942 & 24.78 & 1.292 & 23.84 & 5.822 & 24.45 & 5.822 \\
\hline 129 & 21.5 & 21.05 & 5.915 & 21.5 & 6.138 & 21.66 & 1.348 & 21.2 & 6.014 & 21.24 & 6.014 \\
\hline 130 & 23.9 & 23.73 & 5.783 & 23.64 & 6.001 & 24.08 & 1.304 & 23.35 & 5.88 & 23.49 & 5.88 \\
\hline 131 & 21.6 & 21.58 & 5.67 & 20.69 & 5.884 & 21.76 & 1.281 & 21.12 & 5.765 & 21.36 & 5.765 \\
\hline 138 & 27.1 & 26.86 & 6.256 & 26.91 & 6.492 & 27.3 & 0.552 & 26.5 & 6.361 & 26.67 & 6.361 \\
\hline 139 & 24.9 & 24.23 & 3.003 & 24.9 & 3.116 & 25.08 & 0.541 & 22.58 & 3.053 & 24.23 & 3.053 \\
\hline 140 & 25.5 & 25.5 & 2.875 & 24.74 & 2.983 & 25.69 & 0.518 & 23.79 & 2.923 & 25.45 & 2.923 \\
\hline 141 & 26.4 & 25.95 & 3.094 & 25.85 & 3.211 & 26.59 & 0.558 & 25.26 & 3.146 & 25.95 & 3.146 \\
\hline 142 & 24.6 & 24.6 & 3.315 & 24.55 & 3.44 & 24.78 & 0.655 & 24.03 & 3.371 & 24.6 & 3.371 \\
\hline 143 & 21.5 & 21.01 & 2.911 & 21.16 & 3.021 & 21.66 & 0.525 & 20.81 & 2.96 & 20.86 & 2.96 \\
\hline 144 & 23.9 & 23.23 & 3.003 & 23.9 & 3.116 & 24.08 & 0.541 & 23.33 & 3.053 & 23.09 & 3.053 \\
\hline 145 & 23.2 & 22.99 & 3.132 & 23.11 & 3.25 & 23.37 & 0.565 & 22.25 & 3.185 & 22.81 & 3.185 \\
\hline 146 & 24.8 & 24.16 & 3.395 & 24.8 & 3.523 & 24.98 & 0.597 & 23.71 & 3.452 & 24.16 & 3.452 \\
\hline 147 & 24.7 & 24.63 & 3.492 & 24.58 & 3.624 & 24.88 & 0.617 & 24.13 & 3.551 & 24.26 & 3.551 \\
\hline 148 & 22.6 & 22.55 & 3.458 & 22.6 & 3.588 & 22.77 & 0.603 & 21.88 & 3.516 & 21.81 & 3.516 \\
\hline 149 & 22.1 & 21.95 & 3.365 & 22.06 & 3.492 & 22.26 & 0.592 & 21.72 & 3.422 & 21.26 & 3.422 \\
\hline 150 & 21.6 & 21.58 & 3.51 & 21.6 & 3.642 & 21.76 & 0.998 & 20 & 3.569 & 21.23 & 3.569 \\
\hline
\end{tabular}


Submit or recommend next manuscript to SCIRP and we will provide best service for you:

Accepting pre-submission inquiries through Email, Facebook, LinkedIn, Twitter, etc. A wide selection of journals (inclusive of 9 subjects, more than 200 journals)

Providing 24-hour high-quality service

User-friendly online submission system

Fair and swift peer-review system

Efficient typesetting and proofreading procedure

Display of the result of downloads and visits, as well as the number of cited articles

Maximum dissemination of your research work

Submit your manuscript at: http://papersubmission.scirp.org/

Or contact ajor@scirp.org 